\title{
Adaptación transcultural y validación del test CRAFFT como prueba de pesquisa para consumo problemático, abuso y dependencia de alcohol y otras sustancias en un grupo de adolescentes argentinos \\ Transcultural adaptation and validation of the CRAFFT as a screening test for problematic alcohol and substance use, abuse and dependence in a group of Argentine adolescents
}

Dra. María Carolina Bertini ${ }^{a}$ Dr. Julio Busaniche ${ }^{a}$, Dr. Ferney Baquero, Dr. Alfredo Eymann ${ }^{a}$ Dra. Mariana Krauss ${ }^{a}$, Dra. Marcela Paz ${ }^{a} y$ Dra. Cristina Catsicaris ${ }^{a}$

\section{RESUMEN}

Introducción. El consumo de sustancias de abuso y sus consecuencias son una problemática importante en adolescentes. La pesquisa de consumo problemático, abuso y dependencia (CPAD) de alcohol y otras sustancias debe formar parte de la consulta con adolescentes. El cuestionario POSIT (Problem Oriented Screening Instrument for Teenagers; Pesquisa orientada a los problemas, para adolescentes) y el cuestionario CRAFFT (Car, Relax, Alone, Forget, Family/Friends, Trouble; Auto, relax, solo, olvido, amigos/familia, problema; CRAFFT, por sus siglas en inglés) son para tal fin. POSIT: 17 ítems corresponden al área del uso y abuso de sustancias (POSIT ${ }_{\text {uas }}$ ); ha sido validado en español. CRAFFT: consta de 6 preguntas; no ha sido validado en Argentina. Objetivo. Evaluar la validez de una versión adaptada del CRAFFT al castellano en un grupo de adolescentes argentinos tomando como referencia la pesquisa realizada con POSIT $_{\text {u }}$ Población y métodos. Se realizaron CRAFFT $\mathrm{a}_{\mathrm{a}} \mathrm{y}$ POSIT $_{\text {uas }}$ a adolescentes de 14 a 20 años. Se asignó 1 a la respuesta afirmativa y 0 a la respuesta negativa. Individuo con riesgo aumentado de CPAD, el que puntúe $\geq 2$ en $\mathrm{CRAFFT}_{\mathrm{a}}$ y en POSIT

a. Sección

Adolescencia.

Servicio de

Clínica Pediátrica.

Departamento de

Pediatría. Hospital

Italiano de Buenos

Aires. Argentina.

Correspondencia:

Dra. María Carolina

Bertini: maria.bertini@

hiba.org.ar

Conflicto de intereses:

Ninguno que declarar.

Recibido: 17-6-2014

Aceptado: 14-11-2014
Resultados. Se incluyeron 286 adolescentes; $52 \%$ fueron mujeres; edad media: 16,6 años. $29 \%$ obtuvo puntaje CRAFFT $\geq 2$, y $37 \%$, puntaje POSIT $_{\text {uas }} \geq 2$. Sensibilidad del CRAFFT: $59 \%$; y especificidad: $88 \%$. Valor predictivo positivo CRAFFT: 0,$74 ; y$ valor predictivo negativo: 0,78. El área bajo la curva fue 0,73 . En la regresión lineal, el $\mathrm{R}^{2}$ para las 6 preguntas CRAFFT fue 0,60 . Sexo y edad no modifican los resultados. El alfa de Cronbach fue 0,64. del $59 \%$ y la especificidad, del $88 \%$, comparadas con POSIT uas para pesquisar CPAD de alcohol y otras drogas en adolescentes de nuestro medio. Palabras clave: adolescente, detección de abuso de sustancias, trastornos relacionados con sustancias.

http:/ /dx.doi.org/10.5546/aap.2015.114
Conclusión. La sensibilidad del CRAFFT fue

\section{INTRODUCCIÓN}

El consumo de sustancias de abuso y sus consecuencias son una problemática importante en adolescentes. Según la Quinta Encuesta Nacional a Estudiantes de Enseñanza Media, el abuso de alcohol entre los adolescentes es el principal problema de consumo en nuestro país. También es preocupante el incremento sostenido en el consumo de marihuana por los adolescentes en los últimos años. ${ }^{1}$

El consumo de sustancias psicoactivas en la adolescencia provoca daños en la salud psicofísica, aun antes de que se desarrolle una adicción. ${ }^{2}$ Además, es una etapa de neurodesarrollo y las drogas pueden producir un impacto en el sistema de recompensa cerebral. A menor edad de inicio, mayor es la probabilidad de que se desarrolle un desorden (consumo problemático, abuso o dependencia) en el consumo de sustancias. $^{2}$

Los pediatras tenemos un rol importante en la prevención, detección y manejo de los problemas en la salud integral del adolescente. ${ }^{2}$ Los estudios muestran que, si nos basamos en nuestra propia impresión, tendemos a subestimar el riesgo de los problemas relacionados con el consumo de sustancias psicoactivas en nuestros pacientes. ${ }^{3,4}$

La utilización de instrumentos estructurados de pesquisa durante 
la consulta mejora la detección de desórdenes en el consumo de sustancias en adolescentes. Se han desarrollado numerosos instrumentos de pesquisa; los más utilizados en adolescentes son el POSIT y el CRAFFT.

El POSIT es un cuestionario que busca detectar problemas específicos en distintas áreas de funcionamiento del adolescente. Para la pesquisa de consumo problemático, abuso y dependencia de sustancias, corresponden 17 preguntas $\left(\right.$ POSIT $_{\text {uas }}$ ) (Anexo 1, en formato electrónico). Se administra en forma escrita y ha sido validado en inglés y en español. ${ }^{5-7}$

El CRAFFT es un instrumento de pesquisa para evaluar el riesgo de consumo problemático, abuso o dependencia de alcohol y otras drogas en adolescentes. Su nombre es un acrónimo de 6 palabras claves que componen la prueba: car (auto), relax (relax), alone (solo), forget (olvidos), family and friends (familia y amigos), trouble (problemas) (Anexo 2, en formato electrónico).

Teniendo en cuenta que el CRAFFT no está adaptado ni validado en Argentina, surgió la iniciativa de adaptarlo al castellano y comprobar su validez para detectar consumo problemático, abuso y dependencia de alcohol y otras drogas en adolescentes de nuestro medio.

\section{OBJETIVO}

Evaluar la validez de una versión adaptada del CRAFFT al castellano en un grupo de adolescentes argentinos tomando como referencia la pesquisa realizada con POSIT $_{\text {uas }}$.

\section{POBLACIÓN Y MÉTODOS}

Diseño: estudio observacional de corte transversal.

Población: adolescentes argentinos de entre 14 y 20 años de edad que asistieron a consulta programada o espontánea a la Sección de Adolescencia del Hospital Italiano de Buenos Aires entre octubre de 2011 y febrero de 2013, momento en el que se consiguió el tamaño muestral calculado.

Tamaño muestral: para una población adolescente calculada en 5000 individuos que asisten anualmente al servicio, una prevalencia estimada de consumo de alcohol de $27 \%$ y un margen de error de $5 \%$, se calculó un tamaño muestral de 280 pacientes.

Criterios de exclusión: adolescente con retraso madurativo o retardo mental, que no hable o no entienda el idioma español, o con trastorno psiquiátrico que impida la participación en el estudio.
Adaptación transcultural: el cuestionario CRAFFT consta de una primera parte A de tres preguntas sobre consumo de alcohol, marihuana y otras drogas en los últimos 12 meses. Si una de estas tres son respondidas positivamente, se realiza la parte $\mathrm{B}$ del cuestionario, de seis preguntas que se responden por sí o no (Anexo 2, en formato electrónico).

Previa comunicación con los autores del cuestionario (Dr. Knight y cols.) ${ }^{4}$, a quienes solicitamos autorización para la utilización del CRAFFT en este estudio, se inició la traducción inicial al español (argentino) de forma independiente por parte de un traductor matriculado y otro bilingüe. Una vez que contamos con las dos traducciones directas, se

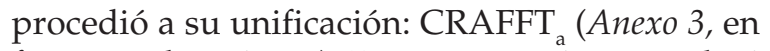
formato electrónico). De esta versión, se realizó una retrotraducción al inglés por dos traductores independientes. Estas versiones en inglés fueron unificadas y comparadas con la versión original, y la final fue avalada por los autores originales.

Se realizó una prueba del CRAFFT a una muestra de 15 adolescentes para comprobar que se entendiera la formulación de los ítems y para recoger los comentarios que pudieran existir. No fue necesario realizar modificaciones.

Validación: se compararon los resultados de CRAFFT $_{a}$ con POSIT $_{\text {uas }}$.

Durante la consulta, el médico informó al adolescente sobre el propósito del presente estudio y le entregó tres hojas: el consentimiento informado (Anexo 4, en formato electrónico), el CRAFFT $_{\text {a }}$ (Anexo 3, en formato electrónico) y el POSIT $_{\text {uas }}$ (Anexo 1, en formato electrónico). Las encuestas fueron autocompletadas y anónimas.

Se le pidió el consentimiento escrito solamente al adolescente (no a los padres ni a los acompañantes) y se respetaron sus derechos de autonomía, privacidad y confidencialidad (Convención sobre los Derechos del Niño, Ley Nacional 26061 y Ley Nacional 26529).

En ambas escalas, se asignó un puntaje de 1 en caso de responder "sí" y 0 si la respuesta era "no". Se consideró como individuo con riesgo de uso problemático, abuso o dependencia de drogas a todo aquel que sumara un total de 2 o más,

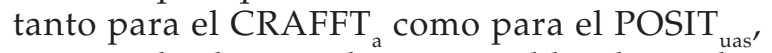
respetando el punto de corte establecido por los autores originales para las dos escalas.

Se calcularon la sensibilidad, la especificidad, el valor predictivo positivo y el valor predictivo negativo del CRAFFT an comparación con el POSIT $_{\text {uas }}$ y el área bajo la curva para un corte 
de 2 preguntas positivas sobre 6 . Se calculó la correlación del CRAFFT con el POSIT $_{\text {uas }}$ con análisis de regresión lineal, que incluyó los seis ítems del CRAFFT; se utilizó alfa de Cronbach para analizar la consistencia interna del CRAFFT.

Análisis estadístico: se realizó con Stata 8.0 (Stata Corporation, Texas, EE.UU.).

Consideraciones éticas: el protocolo de investigación fue aprobado por el Comité de Ética del Hospital Italiano de Buenos Aires el 24 de noviembre de 2011.

\section{RESULTADOS}

En el estudio, se incluyeron 290 adolescentes, de los cuales 286 completaron adecuadamente ambos cuestionarios. Ningún adolescente se negó a participar. El 52\% (148) fueron mujeres. La edad media fue de 16,6 (rango: de 14 a 20) años. El 100\% estaba escolarizado; el 98,6\% (282) cursaba el secundario o lo había terminado; y el 2\% estaba cursando estudios primarios.

El 29\% (83) (IC 23,7-34,3) de los adolescentes tuvo un puntaje igual o mayor a 2 para CRAFFT , $^{\prime}$ y el 37\% (106) (IC 31,4-42,7), para POSIT

La sensibilidad del CRAFFT fue del $59 \%$, y su especificidad, del $88 \%$, comparado con POSIT ${ }_{\text {uas }}$. El valor predictivo positivo para el CRAFFT fue del 0,74 y el valor predictivo negativo, del 0,78 . El área bajo la curva (ROC) con un corte de 2 fue $0,73(0,68-0,79)$, con el $77,3 \%$ de los individuos correctamente clasificados.

En el análisis de regresión lineal, tomando como variable dependiente el puntaje total de POSIT $_{\text {uas }}$ el $R^{2}$ para los seis ítems fue 0,60 . No se modifica con la exclusión de la pregunta 3 (si consume solo) y se modifica poco $(0,58) \sin$ la pregunta 1 (vehículo). Un modelo de 4 preguntas (relajarse, olvidos, familia y amigos, y problemas) tiene casi la misma correlación $\left(R^{2} 0,58\right)$ que las 6 preguntas de CRAFFT en un modelo de regresión lineal (véase la Tabla 1). El sexo y la edad no modifican los resultados.

Analizadas individualmente, la pregunta que mejor relaciona con el POSIT es la pregunta 4 (olvidos), $R^{2}=0,33$, seguida por la pregunta 5 (familia y amigos), $\mathrm{R}^{2}=0,27$.

La consistencia interna del test medida con alfa de Cronbach fue 0,64.

TABLA 1. Regresión lineal CRAFFT de 6 items/4 items

\begin{tabular}{lccc}
\hline Ítems & $\mathbf{R}^{\mathbf{2}}$ & $\mathbf{R}^{2}$ ajustado & Desvío estándar \\
\hline 6 & 0,5902 & 0,5892 & 1,0593 \\
4 & 0,5823 & 0,5762 & 1,0762 \\
\hline
\end{tabular}

\section{DISCUSIÓN}

En nuestro estudio, hemos validado el CRAFFT en un grupo de adolescentes argentinos, luego de realizar la adaptación transcultural del cuestionario.

En este trabajo, hemos comparado el CRAFFT con el POSIT uas' como lo hicieron los autores originales, ya que este último es una herramienta de pesquisa para consumo problemático, abuso y dependencia de alcohol y otras drogas en adolescentes validada al castellano. ${ }^{5-7}$ Knight y cols. también utilizaron una entrevista estructurada diagnóstica de acuerdo con los criterios del DSM-IV de abuso y dependencia de alcohol y otras sustancias para la validación del CRAFFT. ${ }^{8}$ Nosotros no hemos podido utilizar la entrevista psiquiátrica como referencial.

En la utilización del CRAFFT (Anexo 1, en formato electrónico), se sugiere que, si la parte A es negativa (es decir, todas las respuestas se contestan por no), se pasa a la pregunta 1 de la parte B para detectar adolescentes que estuvieran en riesgo de subirse a un vehículo conducido por una persona bajo efectos de sustancias. ${ }^{9}$ Si bien recomendamos utilizar este esquema en la consulta habitual, para el trabajo, decidimos obviarlo y que solo los participantes que habían contestado por sí una pregunta de la parte A respondieran la parte B (dejando afuera del trabajo a los que habían contestado por no todas las preguntas de la parte A). Es decir, que incluimos a los adolescentes que habían consumido sustancias en los últimos 12 meses. El objetivo del trabajo es evaluar el constructo de las seis preguntas de la parte B del CRAFFT ara detectar consumo problemático, abuso y dependencia, y calcular la sensibilidad, especificidad, y determinar su coherencia interna.

La Academia Americana de Pediatría recomienda que preguntemos sobre el consumo de sustancias psicoactivas, incluidos alcohol, tabaco y otras drogas, a nuestros pacientes adolescentes, al menos, una vez al año y que aquellos que hayan consumido alguna sustancia (alcohol u otras drogas de abuso) sean pesquisados con una herramienta validada, como el CRAFFT, para determinar el riesgo de consumo problemático, abuso o dependencia. ${ }^{2}$

El riesgo aumentado (puntaje mayor o igual a 2) del CRAFFT sugiere al pediatra que el paciente requiere una evaluación más completa y detallada sobre el consumo de sustancias, evaluación que determinará la conducta por seguir: consejería, intervención breve, interconsulta o derivación a Salud Mental o a tratamiento especializado. ${ }^{8}$ 
Las ventajas del CRAFFT, comparado con otras pruebas de pesquisa, como AUDIT (Alcohol Use Disorders Identification Test), CAGE (Cut, Annoyed, Guilty, Eye-opener; cuestionario para alcoholismo) y MAST (Michigan Alcoholism Screening Test), son que puede ser realizado verbalmente, no presenta diferencias respecto a género o etnia, es útil para otras drogas distintas al alcohol y está especialmente diseñado para población adolescente. ${ }^{10-12}$ En comparación con el POSIT, el CRAFFT es más breve y puede ser realizado en forma oral.

La relación entre un instrumento y el contexto cultural donde se ha desarrollado responde a ciertos parámetros específicos, como ser los hábitos, las costumbres y las creencias de una cultura que condicionan en gran medida cómo preguntar, cuáles son los términos más apropiados, el sentido de las palabras y de las frases. $^{13}$

Existe una versión en español del CRAFFT llamada CARLOS, ${ }^{14}$ pero no ha sido validada en Argentina. Nosotros elegimos partir de la versión original (en inglés) y realizar nuestra propia adaptación transcultural, siguiendo los pasos internacionalmente consensuados. ${ }^{15}$

En nuestro estudio, el 29\% de los adolescentes que consumieron alcohol, marihuana $u$ otras drogas en los últimos 12 meses presentó un CRAFFT $_{a}$ con riesgo aumentado de consumo problemático, abuso o dependencia de alcohol u otras drogas, porcentaje similar a los reportados por Knight y cols., en el que un $25 \%$ de los participantes presentaron un puntaje de CRAFFT mayor o igual a $2 .^{8}$

En nuestro estudio, la sensibilidad del CRAFFT $_{a}(59 \%)$ fue menor a la comunicada por sus autores originales (80 y $92 \%$ ). La especificidad $(88 \%)$ fue similar a las reportadas por ellos $(82$ y $86 \%)$. Los valores predictivos positivo $(0,74)$ y negativo $(0,78)$ fueron algo menores que los reportados por otros estudios $(0,83$ y 0,91$)$. La consistencia interna del test $(0,64)$ fue similar a la comunicada por los autores originales $(0,68),{ }^{8,16}$

Creemos que la menor sensibilidad del CRAFFT $_{a}$ comparada a la descrita por los autores originales para el CRAFFT se puede deber a que son diferentes poblaciones y a que las preguntas referentes a "vehículo" y "consumir solo" tuvieron poca influencia en la pesquisa. Probablemente, esto último se deba a que, en Argentina, la licencia para conducir se obtiene a mayor edad que en otros países donde fue estudiado el CRAFFT y el consumo de sustancias psicoactivas en adolescentes se suele realizar en grupo. ${ }^{1}$

El CRAFFT a presentó una buena correlación con el $\operatorname{POSIT}_{\text {uas }}\left(\mathrm{R}^{2}=0,60\right)$ en la regresión lineal, aunque algo menor que la reportada por los autores originales $\left(R^{2}=0,68\right)$. Ellos tampoco encuentran influencia del sexo y de la edad en la validez del instrumento. ${ }^{8}$

Con respecto al punto de corte, nosotros también encontramos mejor sensibilidad, especificidad y mejor área bajo la curva (ROC) con dos preguntas positivas del CRAFFT ${ }_{a^{\prime}}$ al igual que Knight y cols. ${ }^{8}$

En nuestro estudio, un modelo de 4 preguntas (relajarse, olvidos, familia y amigos, y problemas) tiene casi la misma correlación que las 6 preguntas en un modelo de regresión lineal $(0,58$ vs. 0,60$)$. Por lo tanto, en la población estudiada, las preguntas referentes a "vehículo" y "consumir solo" tuvieron poca influencia en la pesquisa de consumo problemático, abuso y dependencia de alcohol y otras drogas.

En nuestro medio, se podría utilizar, entonces, el modelo de 4 preguntas del CRAFFT (relajarse, olvidos, familia y amigos, y problemas). Queda por analizar la sensibilidad y especificidad de este modelo y definir el punto de corte. También se puede investigar el comportamiento de la inclusión de otras preguntas para mejorar la sensibilidad de la prueba de pesquisa.

Nuestros resultados no pueden generalizarse a toda la población argentina, ya que la población estudiada pertenece al sistema de obra social y medicina privada (prepago) y tiene un nivel de estudios elevado para la media del país. Otros estudios son necesarios para evaluar el

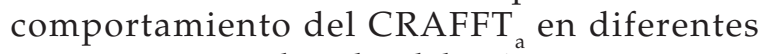
contextos socioculturales del país.

Creemos que el CRAFFT a puede ser incorporado a la entrevista con el adolescente. No reemplaza el interrogatorio personal que cada pediatra pueda hacer con su paciente, sino que lo enriquece, ya que está demostrado que la intuición sola del médico puede subestimar el riesgo. ${ }^{2}$

De acuerdo con nuestro criterio, el CRAFFT puede ser un instrumento válido para ser utilizado por los pediatras o profesionales de la salud que trabajen con adolescentes a fines de pesquisar problemas relacionados con el consumo de alcohol y otras drogas, aun cuando tenga una sensibilidad menor a la reportada. Su fácil realización favorece su inclusión en la consulta con el adolescente. 
Otros estudios son necesarios para evaluar el comportamiento del CRAFFT $a$ en diferentes contextos socioculturales del país.

\section{CONCLUSIÓN}

En este trabajo, hemos validado una versión adaptada del CRAFFT al castellano en un grupo de adolescentes argentinos, tomando como referencia la pesquisa realizada con POSIT $_{\text {uas }}$.

La sensibilidad del CRAFFT fue de $59 \%$, y la especificidad, del $88 \%$, comparada con el POSIT $_{\text {uas }}$. El valor predictivo positivo para el CRAFFT fue del 0,74 y el valor predictivo negativo fue del 0,78 .

\section{REFERENCIAS}

1. SEDRONAR, Observatorio Argentino de Drogas. Quinta Encuesta Nacional a Estudiantes de Enseñanza Media 2011. Informe final de resultados. Buenos Aires: SEDRONAR; 2012. Disponible en: http://www.observatorio.gov.ar/ investigaciones/Quinta\%20Encuesta\%20Nacional\%20 a\%20Estudiantes\%20de\%20Enseñanza\%20Media\%202011. pdf. [Acceso: 4 de marzo de 2013].

2. Committee on Substance Abuse, Levy SJ, Kokotailo PK. Substance use screening, brief intervention, and referral to treatment for pediatricians. Pediatrics 2011;128(5):e1330-40.

3. LevyS,KnightJR. Office-Based Management of Adolescent Substance Use and Abuse. En Neinstein LS, Gordon CM, Katzman DK, Rosen DS, Woods ER, eds. Adolescent Health Care: A Practical Guide. $5^{\text {th }}$ ed. Philadelphia, EE. UU.: Lippincott Williams \& Wilkins; 2008. Págs. 949-58.

4. Wilson CR, Sherritt L, Gates E, Knight JR. Are clinical impressions of adolescent substance use accurate? Pediatrics 2004;114(5):e536-40.

5. Rahdert ER. Problem Oriented Screening Instrument for Teenagers (POSIT). En: Rahdert ER, ed. The Adolescent assessment/referral system manual. Rockville, MD: National Institute on Drug Abuse; 1991. Págs. 67-8.

6. Knight JR, Goodman E, Pulerwitz T, DuRantRH. Reliability of the Problem Oriented Screening Instrument for Teenagers (POSIT) in adolescent medical practice. J Adolesc Health 2001;29(2):125-30.
7. Mariño MC, González Forteza C, Andrade P, Medina Mora ME. Validación de un cuestionario para detectar adolescentes con problemas por el uso de drogas. Salud Ment 1998;21(1):27-36.

8. Knight JR, Sherritt L, Shrier LA, Harris SK, et al. Validity of the CRAFFT substance abuse screening test among adolescent clinic patients. Arch Pediatr Adolesc Med 2002;156(6): 607-14.

9. Center for Adolescent Substance Abuse Research. The CRAFFT Screening Interview. Boston: Children's Hospital Boston; 2009. Disponible en: http: / / www.ceasar-boston. org/CRAFFT/pdf/CRAFFT_English.pdf. [Acceso: 20 de junio de 2011].

10. Knight JR, Sherritt L, Harris SK, Gates EC, et al. Validity of brief alcohol screening tests among adolescents: a comparison of the AUDIT, POSIT, CAGE, and CRAFFT. Alcohol Clin Exp Res 2003;27(1):67-73.

11. Kelly TM, Donovan JE, Chung T, Bukstein OG, et al. Brief screens for detecting alcohol use disorder among 18-20 year old young adults in emergency departments: comparing AUDIT-C, CRAFFT, RAPS4-QF, FAST, RUFT-Cut, and DSM-IV 2-Item Scale. Addict Behav 2009;34(8):668-74.

12. Cook RL, Chung T, Kelly TM, Clark DB. Alcohol screening in young persons attending a sexually transmitted disease clinic. Comparison of AUDIT, CRAFFT, and CAGE instruments. J Gen Intern Med 2005;20(1):1-6.

13. Pane S, Solans M, Gaite L, Serra-Sutton V, et al. Instrumentos de calidad de vida relacionada con la salud en la edad pediátrica. Revisión sistemática de la literatura: actualización. Barcelona: Agencia de Evaluación de Tecnología e Investigación Médicas; 2006. Disponible en: http://www.pediatrasandalucia.org/ Docs/CalidadVidaPediatrico.pdf. [Acceso: 20 de agosto de 2014].

14. Center for Adolescent Substance Abuse Research. Las Preguntas CARLOS(CRAFFT). Boston:Children's Hospital Boston; 2009. Disponible en: http:/ / www.ceasar-boston. org/CRAFFT/pdf/CRAFFT_SA_Spanish.pdf. [Acceso: 20 de agosto de 2014]

15. Arribas A. Adaptación transcultural de instrumentos. Guía para el proceso de validación de instrumentos tipo encuestas. Rev Asoc Med Bahia Blanca 2006;16(3):74-82.

16. Knight JR, Shrier LA, Bravender TD, Farrell M, et al. A new brief screen for adolescent substance abuse. Arch Pediatr Adolesc Med 1999;153(6):591-6. 


\section{ANEXO 1. CUESTIONARIO DE PESQUISA DE PROBLEMAS EN ADOLESCENTES (POSIT ${ }_{\text {uas }}$ )}

El propósito de estas preguntas es ayudarnos a conocer la mejor forma de ayudarte. Por esto, trata de contestar las siguientes preguntas con franqueza.

Este no es un examen; no hay respuestas correctas o incorrectas. Todas las respuestas serán confidenciales.

Contesta todas las preguntas. Si alguna de ellas no se aplica exactamente a ti, escoge la respuesta que más se acerque a la verdad en tu caso. Por favor, marca una " $X$ " sobre tu respuesta.

Si no comprendes alguna palabra, pide ayuda a la persona encargada.

¡Gracias!

1. ¿Te metes en problemas porque consumes drogas o bebidas alcohólicas en la escuela? Sí_ No_

2. ¿Te has hecho daño o le has hecho daño a otra persona accidentalmente estando bajo el efecto del alcohol o de drogas?

Sí__ No_

3. ¿A veces no puedes participar en actividades porque ya no tienes dinero por habértelo gastado en drogas o bebidas alcohólicas?

Sí_ No_

4. ¿Sientes a veces que eres adicto(a) al alcohol o a las drogas?

Sí_ No_

5. ¿Has comenzado a consumir mayor cantidad de drogas o alcohol para obtener el efecto que deseabas?

6. ¿Te vas a veces de alguna fiesta porque no hay bebidas alcohólicas o drogas?

7. ¿Sientes un deseo constante de consumir bebidas alcohólicas o drogas?

8. ¿Has tenido un accidente automovilístico estando bajo el efecto

del alcohol o de drogas?

9. ¿Se te olvidan las cosas que hiciste mientras estabas consumiendo alcohol o drogas?

10. Durante el mes pasado, ¿has manejado un automóvil estando borracho(a) o drogado(a)?

Sí_ No_

Sí_ No_

Sí_ No_

Sí_ No_

Sí_ No_

¿El uso de alcohol o drogas te produce cambios repentinos de humor, como pasar de estar contento(a) a estar triste o viceversa?

Sí_ No_

Sí_ No_

12. ¿Pierdes días de clase o llegas tarde a la escuela por haber consumido bebidas alcohólicas o drogas?

Sí_ No_

13. ¿Te han dicho tus familiares o amigos que debes reducir el uso de bebidas alcohólicas o drogas?

Sí_ No_

14. ¿Tienes discusiones serias con tus amigos o con miembros de tu familia por el uso que haces de bebidas alcohólicas o drogas?

Sí_ No_

15. Cuando consumes bebidas alcohólicas o drogas, ¿tiendes a hacer cosas que normalmente no harías, tales como desobedecer reglas, violar leyes o llegar tarde a casa? Sí_ No _

16. ¿Tienes dificultades en tus relaciones con alguno de tus amigos debido a las bebidas alcohólicas o drogas que consumes?

Sí_ No_

17. ¿Sientes que a veces no puedes controlar el deseo de consumir bebidas alcohólicas o drogas? 


\section{ANEXO 2. CUESTIONARIO CRAFFT}

\section{The CRAFFT Screening Questions}

Please answer all questions honestly; your answers will be kept confidential.

\section{Part $A$}

During the PAST 12 MONTHS, did you:

1. Drink any alcohol (more than a few sips)?

2. Smoke any marijuana or hashish?

3. Use anvthing else to get high?

"anything else" includes illegal drugs, over the counter and prescription drugs, and things that you sniff or "huff"
No

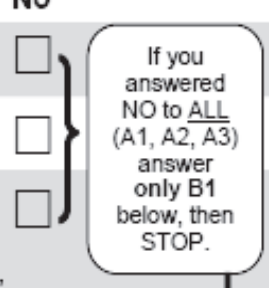

Yes

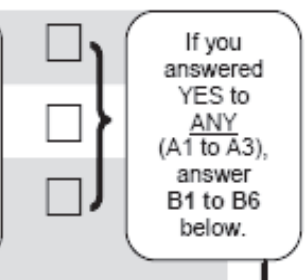

\section{Part B}

No

Yes

1. Have you ever ridden in a CAR driven by someone (including yourself) who was "high" or had been using alcohol or drugs?

2. Do you ever use alcohol or drugs to RELAX, feel better about yourself, or fit in?

3. Do you ever use alcohol or drugs while you are by yourself, or ALONE?

4. Do you ever FORGET things you did while using alcohol or drugs?

5. Do your FAMILY or FRIENDS ever tell you that you should cut down on your drinking or drug use?

6. Have you ever gotten into TROUBLE while you were using alcohol or drugs? 


\section{ANEXO 3. VERSIÓN ARGENTINA DEL CUESTIONARIO CRAFFT: CRAFFTa}

\section{PARTE A}

Durante los últimos 12 meses:

1. ¿Has consumido bebidas alcohólicas (más de unos pocos sorbos)?

Sí_- No_

Sí__ No_

2. ¿Has fumado marihuana?

3. ¿Has usado algún otro tipo de sustancias que alteren tu estado de ánimo o de conciencia?*

* El término "algún otro tipo" se refiere a drogas ilícitas, medicamentos de venta libre o de venta con receta médica, así como a sustancias inhalables que alteren tu estado mental.

(Si respondiste "Sí" a CUALQUIERA de las anteriores 3 preguntas, pasá a las preguntas B1-B6).

\section{PARTE B}

1. ¿Alguna vez viajaste en un vehículo conducido por vos u otra persona que hubiera consumido alcohol o drogas?

2. ¿Alguna vez usaste alcohol o drogas para relajarte, sentirte mejor con vos mismo o para integrarte a un grupo?

3. ¿Alguna vez consumiste alcohol o drogas mientras estabas solo/a?

4. ¿Alguna vez te olvidaste de cosas que hiciste por haber consumido alcohol o drogas?

5. ¿Alguna vez tu familia o amigos te dijeron que disminuyeras el consumo de alcohol o drogas?

Sí_ No_

Sí_ No_

Sí_- No_

Sí_ No_

Sí_ No_

Sí_- No_

6. ¿Alguna vez tuviste problemas por haber consumido alcohol o drogas? 


\title{
ANEXO 4. CONSENTIMIENTO INFORMADO
}

\author{
Buenos Aires, ...... de ............... de 201...
}

1. Queremos invitarte a participar en el estudio "Validación del CRAFFT como prueba de pesquisa para uso y abuso de sustancias en adolescentes argentinos", el cual se está llevando a cabo por parte del personal médico del Servicio de Adolescencia del Hospital Italiano de Buenos Aires.

2. Este estudio busca validar una escala que se usa en otros países del mundo para detectar a aquellos jóvenes de tu edad que pueden estar teniendo problemas con el consumo de alcohol y/o drogas.

3. El cuestionario consta de 6 preguntas, en las cuales debes responder "Sí" o "No" de acuerdo con lo que vos consideres se adecúa más para tu caso. Este cuestionario no es un examen y, por lo tanto, no hay respuestas correctas o incorrectas.

4. El cuestionario debe ser completado de forma individual (sin ayuda de tus padres, hermanos, pareja, etc.) y anónima. No es necesario que pongas ningún dato que pueda identificarte.

5. Recordá que, aun teniendo menos de 18 años de edad, tenés derecho a que la información que completes sea confidencial y nadie tiene por qué enterarse, a menos que vos estés de acuerdo. Si vos considerás que tenés problemas con el alcohol y/o las drogas, te ofrecemos hacer una entrevista especializada con el equipo de Salud Mental del Hospital Italiano de Buenos Aires y buscaremos cómo ofrecerte la ayuda que necesites.

6. Antes de que digas que sí quieres participar de este estudio, por favor, preguntá todas las dudas que tengas y nosotros las responderemos. En caso de que surjan dudas adicionales en relación con el estudio, podrás dirigirlas al Dr. Ferney Baquero del Servicio de Adolescencia al correo electrónico ferney.baquero@hospitalitaliano.com.

7. No tenés que participar si no querés; todo depende de vos. Podés decir que sí ahora y luego cambiar de opinión. Lo único que debés hacer, si así lo deseás, es decírnoslo. Nadie se enojará con vos ni dejarás de recibir la atención médica que necesites.

8. Este estudio ha sido evaluado por el Comité de Ética de Protocolos de Investigación del Hospital Italiano (CEPI).

9. Si aceptás participar de este estudio, por favor, firmá en el correspondiente espacio a continuación. Recibirás una copia de este formulario para que vos lo conserves si lo deseás.

Aclaración:

Documento:

Aclaración:

Documento:

(Médico)
Aclaración:

Documento:

(Testigo) 